\title{
Bisection of loudness
}

\author{
EDWARD C. CARTERETTE \\ University of California, Los Angeles, Los Angeles, California 90024 \\ and \\ NORMAN H. ANDERSON \\ University of California, San Diego, La Jolla, California 92093
}

\begin{abstract}
In a loudness bisection task, subjects varied one sound to lie halfway between two given sounds in terms of loudness. The two given sounds were varied from 30 to $90 \mathrm{~dB}$ in a 4 by 9 factorial design. Functional measurement methods based on monotone analysis provided good support for the bisection model, and yielded a loudness scale with an exponent of about .3, except for a falloff at lower intensities. Two other tasks, judging average loudness and difference in loudness of the two given sounds, yielded mixed results. In Experiment 2, in particular, the differencing judgments were not additive, even under monotone transformation. These analyses also indicated that previous applications of monotone analysis have typically lacked adequate power to allow any conclusion about the operative model. Overall, the present bisection scale agrees with Garner's lambda scale, and the present theoretical approach agrees with that of Garner in its emphasis on algebraic models as a foundation for psychological measurement.
\end{abstract}

The quality of loudness has good reasons for being a primary focus of psychophysical measurement. It has enormous dynamic range, in contrast to skin temperature or pressure. It is an energetic, sensory dimension, in contrast to length. It has nearly the ecological importance of brightness, but not nearly the complications from adaptation. And it is experimentally flexible and easy to control. For these and other reasons, loudness has become a primary test case for theories of psychophysical measurement.

The method of bisection is attractive because it rests on sensory comparison and avoids artificial response language. If the subject's bisection response was indeed at the sensory midpoint, then a simple procedure of successive bisection would yield a linear (equal-interval) scale of sensation. Two arbitrary initial stimuli would be called 0 and 100 . Their bisector would then have the value 50 , two further bisections would yield the 25 and 75 points, four further bisections would yield the $12.5,37.5,62.5$,

This work was supported by National Institute of Mental Health Grant MH-07809 to the first author, and by National Science Foundation Grant BMS 74-19124 to the second author. A preliminary report of this work was presented at the XXI International Congress of Psychology, Paris, July 1976. We wish to thank Alan Barnebey for help with apparatus and computing, Cheryl Smart for programming and managing the experiments, Clifford Butzin and Anthony Harris for advice and assistance in data analysis, and Edward Alf for developing the HP-67 program used for fitting power functions. Requests for reprints may be sent to Edward Carterette, Psychology, University of California, Los Angeles, Los Angeles, California 90024, or to Norman Anderson, Psychology C-009, University of California, San Diego, La Jolla, California 92093. and 87.5 points, and so on, to as fine a division as desired. Granted that the bisection response is at the sensory midpoint, these numbers would constitute a linear scale of loudness.

But the method of bisection has long resisted analysis. The successive bisection procedure outlined above rests on the strong assumption that the bisections are at the sensory midpoint. However, order effects, such as are found with loudness, imply that bisections are not at the sensory midpoint. Nor does absence of order effects guarantee the midpoint assumption. For example, Stevens $(1955$, p. 825$)$ has argued that bisection judgments confound difference and ratio comparisons, and that would produce bisection responses systematically different from the sensory midpoint. These and other problems have held back development of the method of bisection.

Developments in functional measurement have provided a general, practicable analysis for bisection (Anderson, 1970, 1976, 1977a; Weiss, 1975). A common and attractive hypothesis is that bisection involves an equating of sense distances. If $s_{1}$ and $s_{2}$ are the subjective or psychological values of the two bisectee stimuli, and $r$ is the psychological value of the bisector response, then the two sense distances are $\left(r-s_{1}\right)$ and $\left(s_{2}-r\right)$. Weights may be included to allow for differential attention to the two sense distances. Hence, the hypothesis of equated sense distances would be written,

$$
w_{1}\left(r-s_{1}\right)=w_{2}\left(s_{2}-r\right),
$$

where $w_{1}$ and $w_{2}$ are the weights. Letting $w=$ 
$w_{2} /\left(w_{1}+w_{2}\right)$ and solving for $r$ yields the simple averaging model:

$$
r=(1-w) s_{1}+w s_{2} .
$$

The weight parameter, $w$, plays a special role. With it, the model does not require the bisection to be at the sensory midpoint. Hence, the model could allow for instructions to make, say, a 70:30 division of the total sense distance. More generally, $\mathrm{w}$ can function as a criterion parameter, and also allow for certain kinds of order effects. Most important for present purposes is that the model analysis makes automatic allowance for the weight parameter.

Equation 1 might seem to be untestable because it deals with unobservables. The investigator knows only the observable physical values of the stimuli and response, whereas the bisection process operates with the unknown psychological values. Without these psychological values, the bisection model might seem beyond test. However, functional measurement provides a test that depends only on the algebraic structure of the model.

The logic is straightforward. It can be assumed that the psychological value of the bisection response, denoted by $r$, is some monotone function of physical value, denoted by $R$. If the bisection model is correct, then some monotone transformation of the observed response will be additive. If the bisectee stimuli are varied in factorial design, then the parallelism theorem will apply. That allows a proper test of the bisection model.

No auxiliary assumptions are necessary. The weight parameter is automatically absorbed into the scale unit so that such order effects are automatically taken into account. The psychological values of the stimuli are derivative from the analysis. The psychophysical law relating $R$ and $r$ is also derivative from the analysis. All that is at issue is the algebraic form of the bisection model. This algebraic form serves as the base and frame for measurement in a truly scalefree way.

Application of this analysis involves three technical problems. The first, that of finding a best possible monotone transformation, may be accomplished using Kruskal's (1965) MONANOVA program or the ADDALS program of de Leeuw, Young, and Takane (1976). The second, that of testing goodness of fit to the bisection model, may be accomplished with a general error theory for nonmetric analysis (Anderson, 1977a, 1977b). If Stevens' hypothesis that bisection involves both difference and ratio comparisons was correct, for example, then the bisection model should fail the test of goodness of fit. The third problem, that of ensuring adequate power in the analysis, presents serious difficulties which are discussed in later sections of this paper.
Nearly all previous studies of the bisection model have rested on special assumptions, that $w=1 / 2$, for example, or that the psychophysical law has some specific form. Moreover, previous studies have generally assumed that the model was correct without providing an adequate test of goodness of fit. A notable exception is the report of Garner (1954a). Garner's approach has been criticized by Stevens $(1955,1957)$ because of order effect problems, and it has fallen into neglect despite its obvious claims to more careful consideration. Indeed, Garner's method is basically correct, as will be seen below, and the present results support those obtained by Garner.

Two experiments are reported here, only one of which studied bisection. The first was a preliminary experiment on judgments of average loudness and of difference in loudness of noise pairs. These same two tasks were used together with the bisection task in the second experiment. These three tasks were included to assess cross-task invariance of the loudness scale in the same way as has been done with heaviness (Anderson, 1972, 1974b) and grayness(Anderson, 1976, 1977b, Figure 7).

\section{EXPERIMENT 1}

\section{Method}

Subjects heard pairs of noise bursts and judged either their average loudness or their difference in loudness using a graphic rating scale in the form of a response keyboard with 21 keys. First and second noises were varied in a 5 by 5 factorial design from 40 to $80 \mathrm{~dB}$ (SPL) in 10-dB steps.

\section{Part 1: Averaging}

Duration of the first and second noises was .5 or $1.25 \mathrm{sec}$, all four duration combinations being employed in a 2 by 2 design. The interstimulus interval was $.5 \mathrm{sec}$, and the trial was terminated by response following of fset of the second noise. If no response was given within a $2.5-\mathrm{sec}$ period, the trial was repeated. The next trial followed the response by $1 \mathrm{sec}$.

In the initial instruction-practice period, low and high endanchor stimuli were presented to set up the stimulus-response coordination (Anderson, 1974a, p. 232; 1974c, p. 245). The low end-anchor was a pair of $30-\mathrm{dB}$ noises; the subject was told that this was the softest sound, corresponding to the leftmost response key. The high end-anchor was a pair of $92-\mathrm{dB}$ noises; the subject was told that this was the loudest sound, corresponding to the rightmost response key. In-between keys were to be used to represent in-between loudnesses in the natural way.

Four replications of the 5 by 5 design were presented for each of the four duration combinations. Each replication was started with a new presentation of the end-anchors and was followed by a short rest. Experimental sessions lasted approximately $40 \mathrm{~min}$. Each of four paid students with normal hearing served for four replications for each of the four duration combinations. These same four subjects also served in both the averaging and differencing tasks in Part 2.

\section{Part 2: Averaging}

Design and procedure were the same as in Part 1, except that both noises had a .5 -sec duration. Eight replications of the 5 by 5 design were presented on each of four sessions. Thus, each subject judged each noise combination a total of 32 times. Each 
replication began with a new presentation of the end-anchors, and a short rest was given halfway through each session.

\section{Part 3: Differencing}

For the differencing task, both first and second noises had .5 -sec duration and were separated by $.25 \mathrm{sec}$. However, each such noise pair was presented twice on each trial with a 1-sec interpair interval. This double presentation was used to help stabilize responding in what seemed to be a somewhat variable task. Other times were the same as for the averaging task.

In the initial instruction-practice period, the subject was told to press the center key when the sounds were equally loud, to press leftward keys when the first sound was louder, and to press rightward keys when the second sound was louder. End-anchors of 92-30 dB and 32-90 dB were presented with instructions that these were the two biggest differences, corresponding to the leftmost and rightmost keys, respectively. In-between keys were to be used to represent in-between loudness differences in the natural way. Each replication of the 5 by 5 design was started with a new presentation of the end-anchors, and a short rest was given every four replications.

Eight replications of the 5 by 5 design were presented on each of four sessions. Thus, each subject judged each noise combination a total of 32 times. This differencing task was run after the above averaging task.

\section{General Procedure}

The experiment was controlled by a Hewlett-Packard computer which presented stimuli according to the prescribed time schedule, recorded responses, and provided initial analyses of the data (see Carterette, Barnebey, Lovell, Nagel, \& Friedman, 1972). The response keyboard was treated as a graphic rating scale with the keys coded 1-21 for response recording. This has been changed to a $0-20$ scale in the figures below. Stimulus combinations were presented in separate randomized order for each replication for each subject.

The noise was obtained from a General Radio Co. Model 1382 noise generator, whose output was led through a Krohn-Hite 315A variable filter set to pass frequencies between 40 and $6,500 \mathrm{~Hz}$, with a 24-dB-per-octave slope at the half-power points. After being routed to a Crown D40 wide-band power amplifier, thence to a digital attenuator, noise bursts having equal rise and fall times of $10 \mathrm{msec}$ were shaped by a Grason-Stadler $829 \mathrm{~S}$ electronic switch. Finally, the noise bursts were delivered binaurally in phase to Koss ESP-9 earphones mounted in circumnaural cushions. Calibrations of absolute overall SPL were made using a Brüel and Kjaer Type 4151 artificial ear fitted with a Type 4144 condensor microphone (25.4-mm diam). Signal levels were read from a Brüel and Kjaer Type 2107 frequency analyzer. All listening was done in an anechoic room which was acoustically isolated from externally generated sound.

\section{Model Analysis}

\section{Results}

Both the averaging and the differencing tasks were expected to follow adding-type models. In fact, the models for both tasks are formally similar to Equation 1. If the rating response is a linear (equal-interval) scale, as is expected from previous work (Anderson, 1974a, 1974c), then the overt response will be a linear function of the implicit response, $r$, of Equation 1 . Hence, if the averaging and differencing models are correct, then the observed data will exhibit parallelism.

The results are in Figure 1. The three panels present factorial plots of mean judgments from the two averaging tasks and from the differencing task. The main characteristic of these data is that they exhibit the expected pattern of parallelism. The five curves are approximately parallel in all three panels of Figure 1. That suggests that simple averaging and differencing rules are operative in these judgments.

Of special interest is the vertical spacing of the five curves in each panel. By virtue of the parallelism, the response measure may be considered to be a linear scale. As a consequence, the vertical elevations of the curves measure the subjective loudness of the first noise on a linear scale.

Loudness is clearly nonlinear in decibels. The vertical distance between adjacent curves represents the loudness increment produced by an interisity increment of $10 \mathrm{~dB}$. The pattern of the curves thus shows that $10 \mathrm{~dB}$ has more than twice the effect at the upper end of the stimulus range than at the lower end.

One purpose of this experiment was to get a pre-

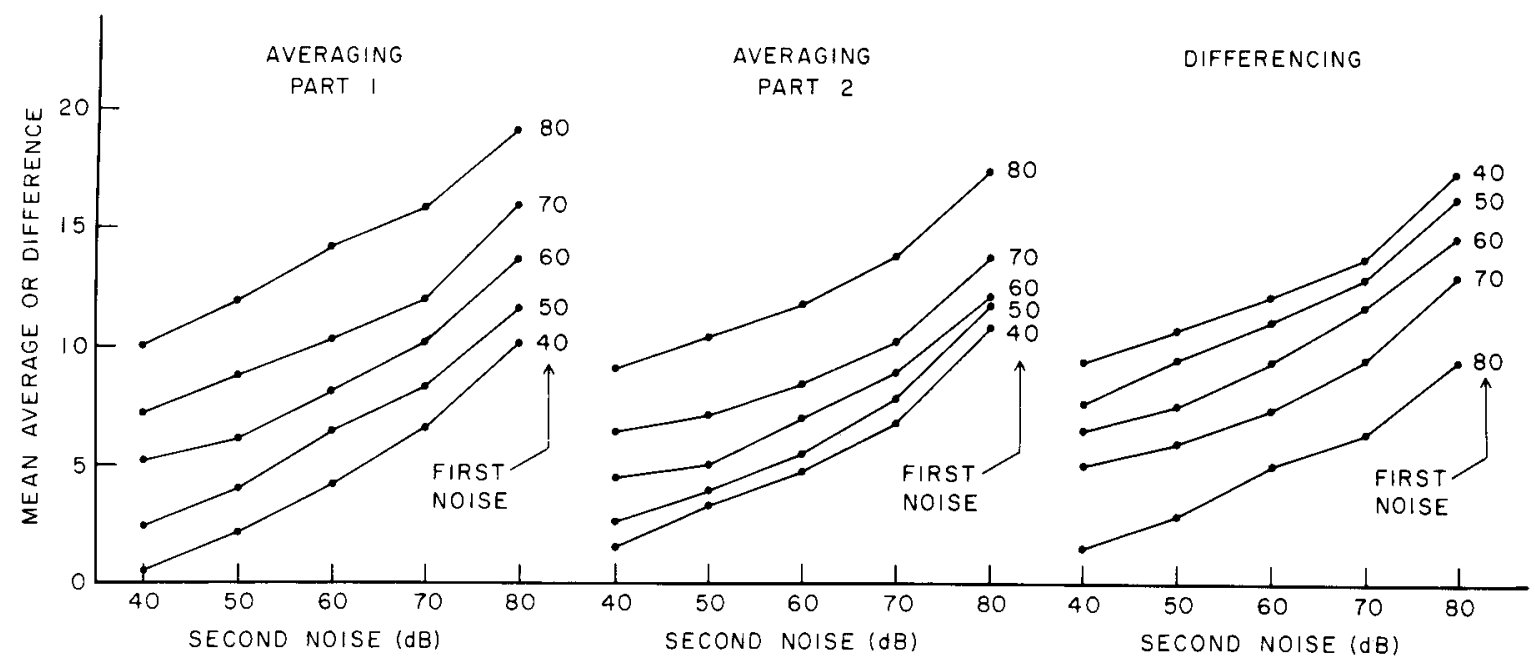

Figure 1. Factorial graphs of mean judgment of average loudness, or difference in loudness, Experiment 1. (Ordering of curves reversed for differencing task in which louder first noises correspond to smaller numbers.) 
liminary loudness scale for use in the bisection task of Experiment 2. Design and analysis will be more informative and efficient if stimuli can be chosen at approximately equal intervals of loudness. By virtue of the parallelism, the row means of the data provide a linear scale of loudness. Accordingly, power functions were fit to the row means of the averaging and differencing data of Part 2 with intensity measured in sound pressure, $P$. Because linear scales have unknown zero points, an additive, zero-point constant must be included in the power function fit. Here and below, therefore, exponents were estimated by fitting the equation, $a+b P^{n}$, where $a$ and $b$ are constants. This yielded exponents of .30 for the averaging task, .35 for the differencing task. On this basis, the intensity levels for Experiment 2 were chosen to be equally spaced on a $\mathrm{P}^{.33}$ scale.

\section{Statistical Analyses}

The different noise durations of Part 1 gave essentially the same results. Neither the main effect nor the interaction of the two durations was significant. Two interactions of duration and intensity were marginally significant, and inspection showed that this reflected a slight tendency to treat the $80-\mathrm{dB}$ sound as louder at the longer, 1.25-sec duration. Accordingly, the .5-sec interval was chosen for use in Part 2 and in Experiment 2.

Goodness of fit for the averaging and differencing models can be tested by the First Noise by Second Noise interaction. If the models are correct, then this interaction is zero in principle and should be nonsignificant in practice. The group analyses supported the models, since the interactions were nonsignificant, $F(16,48)=.72,1.88$, and .73 for the three respective panels of Figure 1.

Individual analyses were also performed for the averaging and differencing tasks of Part 2 . The interaction terms were significant for three of four subjects in the averaging task, mean $F(16,700)=2.24$, and for all four subjects in the differencing task, mean $F(16,700)=7.56$. Inspection showed that the deviations from parallelism were quite small, with no apparent pattern except for certain signs of floorceiling effects in the response scale. Since each individual test was based on a total of 800 responses, even small deviations were significant. Provisionally, at least, these deviations from parallelism do not seem to raise serious questions about the models.

A more serious problem was that two subjects in the averaging task in Part 2 appeared to adopt a semidichotomous valuation rule for the first noise, tending to consider it as either soft or loud. Neither subject had done this in Part 1, and the effect can be seen in Figure 1 as a closer bunching of the lower curves in the middle than in the left panel. This kind of valuation operation does not affect the integration model itself, but it does intrude a nonsensory element into the scaling (see also below).

\section{EXPERIMENT 2}

\section{Method}

Three tasks were used: bisecting, averaging, and differencing. The latter two tasks were the same as in Experiment 1 and virtually identical procedure was used. In the bisecting task, the subject adjusted a variable-intensity noise to lie halfway between two given noises.

\section{Design}

For scaling purposes, the design was changed from a square to a rectangular, 4 by 9 , First Noise by Second Noise factorial. The nine levels of second noise were $30,50,62,68,73,78,83$, 87 , and $90 \mathrm{~dB}$. These were chosen to lie at equal intervals on a $\mathrm{P}^{33}$ scale in accord with the results of Experiment 1 . The marginal means of this nine-level factor were intended to form the basis for scaling. The four levels of first noise were $50,68,78$, and $87 \mathrm{~dB}$, also at equal intervals on the $P^{.33}$ scale. The four levels of the first factor allow monotone transformation and parallelism analysis while keeping the design manageably small.

Eight paid subjects served one practice session and then for 10 replications of the 4 by 9 design for each of the three tasks. One subject gave disordinal data in the bisection task, and his data are not considered here. Each replication was begun with presentation of the two end-anchors, followed by a newly randomized sequence of the 36 experimental trials.

\section{Averaging and Differencing}

Trial timing and general procedure were the same as in Part 2 of Experiment 1. The low and high end-anchors for the averaging task were noise pairs of 30 and $92 \mathrm{~dB}$, respectively. Corresponding end-anchors for the differencing task were the $92-30$ and $30-92 \mathrm{~dB}$ pairs.

\section{Bisecting}

In this task, the unit stimulus presentation was a sequence of three noise bursts, of which the first and last were specified by the 4 by 9 factorial design. The middle noise was variable, and the subject could leave it unchanged or press a key to raise or lower it $1 \mathrm{~dB}$ on each presentation. The next presentation began when this key was pressed or else $1.25 \mathrm{sec}$ later. This process was repeated until the subject was satisfied, whereupon a press on another key signaled the computer to record the response information and begin the next trial. Duration of each noise burst was $.5 \mathrm{sec}$ with interburst intervals of $.5 \mathrm{sec}$. The intertrial interval was $1.25 \mathrm{sec}$. The end-anchor combinations from the averaging task were also used to reaffirm the total loudness range at the beginning of each replication.

The initial intensity of the middle noise on any trial was set halfway between the two given noises on the $P^{.33}$ scale plus a random number of decibels. The random number was $\pm 1, \pm 2$, and \pm 3 with equal frequency. This procedure kept adjustment time small and still balanced for possible order effects.

\section{Single Stimulus Judgments}

In the final session of the experiment, subjects judged the loudness of each of the nine single stimuli using two response methods, rating and magnitude estimation. The rating method used the response keyboard. With magnitude estimation, the 73-dB noise was called 100 and other noises were to be assigned numbers relative to 100 in the standard manner. A total of 8 and 32 replications of the nine basic stimuli were presented in separate randomized order for the rating and magnitude estimation responses, repectively.

\section{Bisection \\ Results}

Since the bisection response is measured on the physical intensity scale, it must be transformed to the 


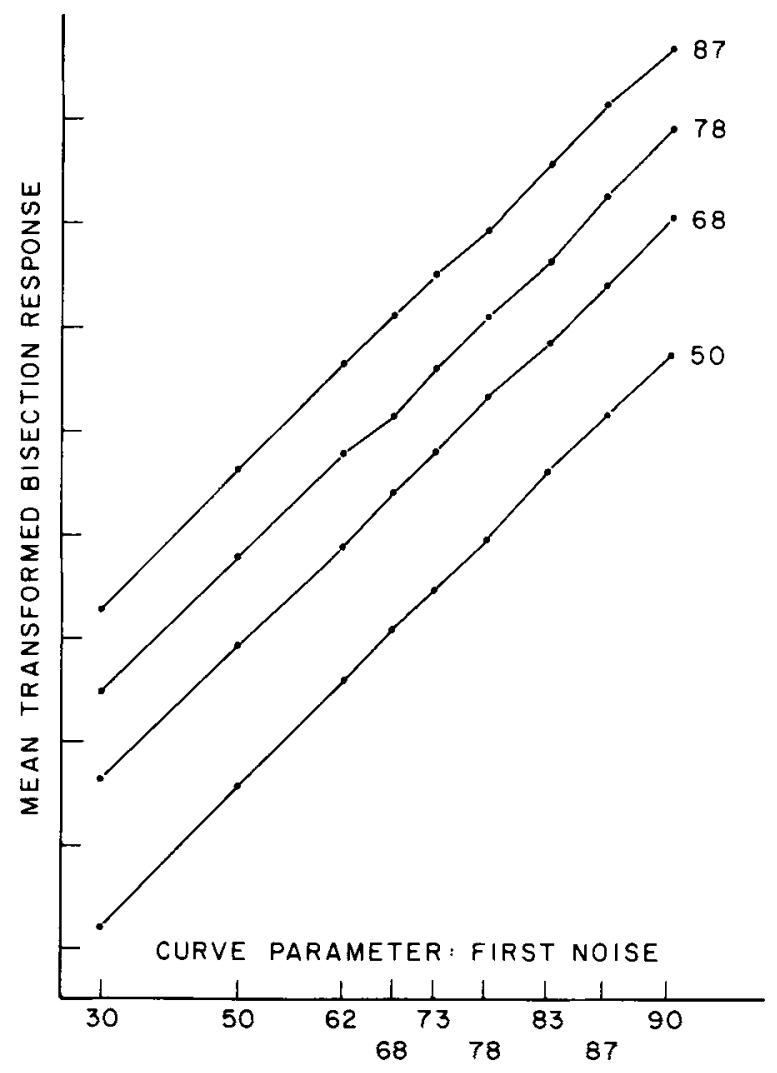

SECOND NOISE ( $A B$ ): FUNCTIONAL- SCALE SPACING

Figure 2. Mean transformed bisection data, group data.

psychological sensation scale. The technical problems of transformation are important but somewhat complicated and accordingly are deferred to a later section. The following discussion considers the transformed bisection data. These data are on a linear scale, so that the zero point is arbitrary. For present purposes, however, no more is necessary.

Figure 2 shows the factorial plot of the mean transformed bisection data. Noise levels are spaced on the horizontal axis at their functional scale values. If the bisection model is correct, then the four curves should be both straight and parallel. As can be seen, they are very nearly straight and very close to parallel.

A different graphical format is used in Figure 3, which also presents results for the individual subjects. The upper-left panel replots the data of Figure 2, but now the stimuli are spaced on the horizontal axis according to the .33 power of sound pressure. Since the noise intensities were chosen to be equidistant on this scale, these curves would be straight lines if loudness were indeed a .33 power of sound pressure. The curves are straight over the upper range, although there is a dropoff at the two lowest intensities This dropoff agrees with previous work and is sometimes considered to reflect the need for a threshold constant, an important determiner of curve shape at lower intensities (e.g., Luce \& Galanter, 1963, p. 281; McGill, 1960).
All subjects showed essentially the same pattern. The four curves in each panel of Figure 3 are approximately parallel, and that supports the bisection model at the individual level. Further, curve shape is very uniform across subjects, which means that they all had nearly the same psychophysical function for loudness.

Table 1 presents the mean values of the raw and the transformed bisection responses. The raw data, in the upper half of the table, are markedly nonparallel showing steady convergence as the intensity of the second noise increases. Thus, the range in the first column is from 43.0 to $63.3 \mathrm{~dB}$, a net difference of $20.3 \mathrm{~dB}$. The range in the last column is only $11.8 \mathrm{~dB}$. This nonparallelism simply reflects the fact that the physical decibel scale is not a linear scale of loudness.

The lower half of Table 1 presents the mean values of the transformed response. The zero and unit of this scale are arbitrary, of course, and were chosen for convenience. The top four rows represent the same values as were plotted in the four corresponding curves of Figure 2. These data are very nearly parallel,

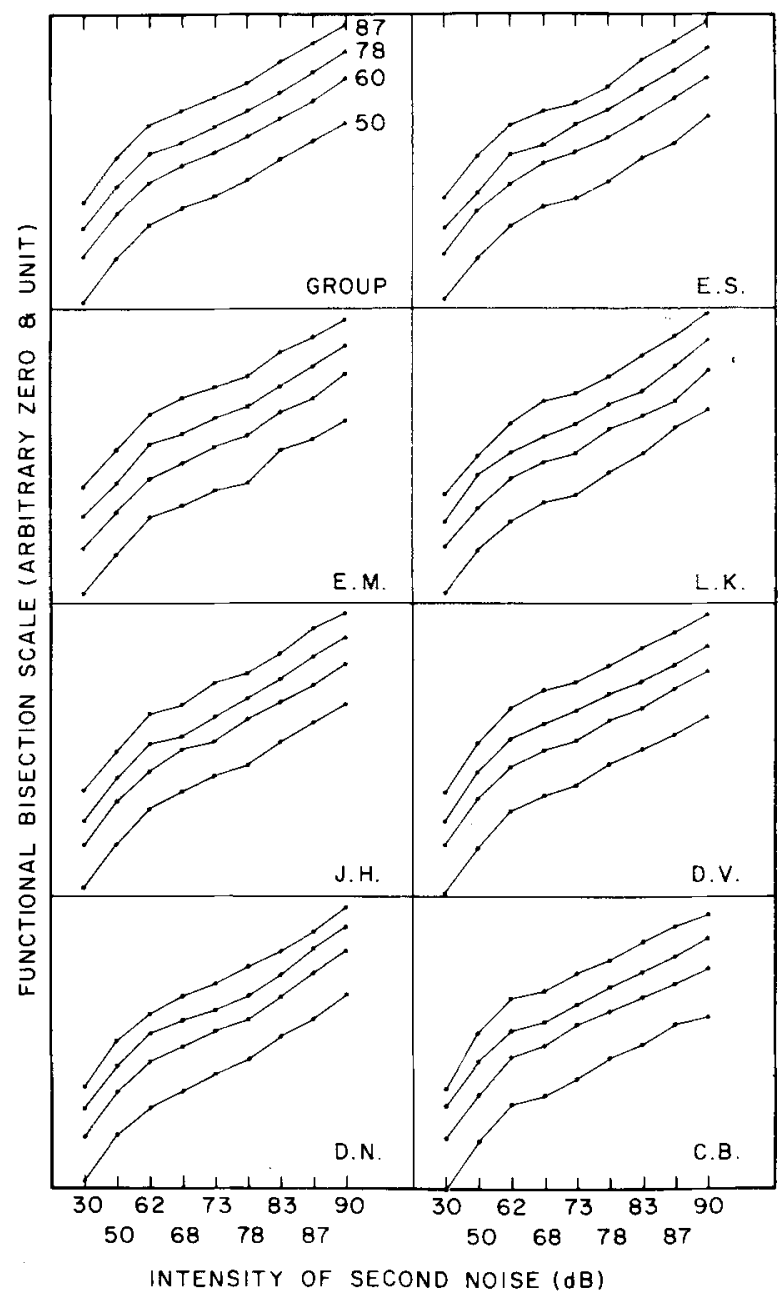

Figure 3. Transformed bisection data for individuals and groups. 
Table 1

Mean Raw and Transformed Bisection Responses

\begin{tabular}{|c|c|c|c|c|c|c|c|c|c|}
\hline \multirow{2}{*}{$\begin{array}{l}\text { First (Row) } \\
\text { Intensity }\end{array}$} & \multicolumn{9}{|c|}{ Second (Column) Intensity (dB) } \\
\hline & 30 & 50 & 62 & 68 & 73 & 78 & 83 & 87 & 90 \\
\hline & \multicolumn{9}{|c|}{ Raw Response } \\
\hline $\begin{array}{l}50 \\
68 \\
78 \\
87\end{array}$ & $\begin{array}{l}43.0 \\
52.9 \\
58.0 \\
63.3\end{array}$ & $\begin{array}{l}52.1 \\
61.0 \\
66.2 \\
71.2\end{array}$ & $\begin{array}{l}58.3 \\
66.7 \\
71.9 \\
76.8\end{array}$ & $\begin{array}{l}61.8 \\
69.8 \\
73.8 \\
79.4\end{array}$ & $\begin{array}{l}64.3 \\
71.9 \\
76.5 \\
81.3\end{array}$ & $\begin{array}{l}67.3 \\
75.0 \\
79.4 \\
82.9\end{array}$ & $\begin{array}{l}71.1 \\
77.9 \\
81.5 \\
85.4\end{array}$ & $\begin{array}{l}74.3 \\
80.7 \\
84.4 \\
87.6\end{array}$ & $\begin{array}{l}77.3 \\
83.6 \\
86.9 \\
89.1\end{array}$ \\
\hline \multirow[t]{2}{*}{ Mean } & 54.3 & 62.6 & 68.4 & 71.2 & 73.5 & 76.2 & 79.0 & 81.8 & 84.2 \\
\hline & \multicolumn{9}{|c|}{ Transformed Response } \\
\hline $\begin{array}{l}50 \\
68 \\
78 \\
87\end{array}$ & $\begin{array}{r}.80 \\
2.05 \\
2.79 \\
3.50\end{array}$ & $\begin{array}{l}2.00 \\
3.20 \\
3.93 \\
4.69\end{array}$ & $\begin{array}{l}2.89 \\
4.03 \\
4.80 \\
5.58\end{array}$ & $\begin{array}{l}3.32 \\
4.49 \\
5.13 \\
5.98\end{array}$ & $\begin{array}{l}3.65 \\
4.83 \\
5.54 \\
6.31\end{array}$ & $\begin{array}{l}4.08 \\
5.29 \\
5.97 \\
6.70\end{array}$ & $\begin{array}{l}4.67 \\
5.76 \\
6.43 \\
7.25\end{array}$ & $\begin{array}{l}5.14 \\
6.23 \\
6.99 \\
7.75\end{array}$ & $\begin{array}{l}5.65 \\
6.81 \\
7.55 \\
8.23\end{array}$ \\
\hline Mean & 2.29 & 3.45 & 4.32 & 4.73 & 5.08 & 5.51 & 6.03 & 6.53 & 7.06 \\
\hline
\end{tabular}

although some trace of convergence still remains. ${ }^{1}$ Thus, the range between the top and bottom rows decreases slightly from 2.70 in the first column to 2.58 in the last column. In more familiar terms, the mean magnitude discrepancy from parallelism, averaged over the 36 cells of the design, had a decibel equivalent of $.14 \mathrm{~dB}$.

A formal statistical test of the bisection model is given by the interaction test from the analysis of variance (see below). This was marginally significant for four of the seven subjects (E.S., L.K., J.H., D.N.), and their graphs in Figure 3 show a slight tendency to converge to the right, as already noted in the group data in Table 1. The deviations from parallelism are quite small, however, and accounted for less than $.2 \%$ of the systematic variance for any subject. Provisionally, at least, it seems reasonable to conclude that the bisection model is basically correct.

\section{Loudness Scaling}

The bisection scale of loudness is given by the marginal means of the factorial table of transformed data. This is the bottom row in Table 1. In graphical terms, this scale can be viewed as the mean of the four curves in Figure 2. If the bisection model is correct, then this mean curve provides a linear (equalinterval) scale of loudness.

This loudness scale is plotted as a function of decibel intensity in Figures 4 and 5 for the group and the individuals. These curves show steady acceleration, and grow increasingly convex as intensity increases. Except at the highest intensities, these loudness scales are very similar across individuals.

This bisection scale of loudness disagrees with results obtained from the method of magnitude estimation. Considered as a function of sound pressure, magnitude estimation generally yields a power function exponent around .6. Bisection yields an exponent around .33 , as already noted in the dis- cussion of Figure 3. To check this exponent, a power function was fit to the bisection scale in the bottom row of Table 1, the two lowest points being omitted. Since the bisection scale is a linear scale with arbitrary zero, the fitted function had the form $a+b P^{n}$ where $a$ is a zero-point constant. This fit yielded the nearly identical exponent of .35 .

This disagreement between the bisection scale of loudness and results from magnitude estimation was expected, of course, since the .33 exponent was obtained initially from the rating data of Experiment 1. It is well known that ratings and magnitude estimation disagree on most stimulus dimensions, with exponents obtained from rating data running modally

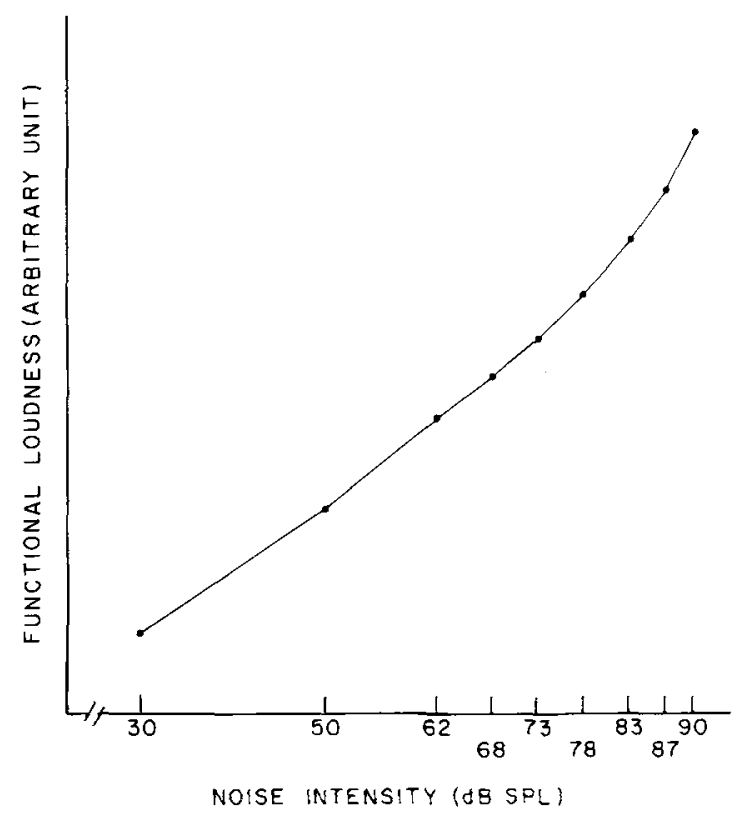

Figure 4. Functional loudness scale from bisection plotted against noise level in decibels. 


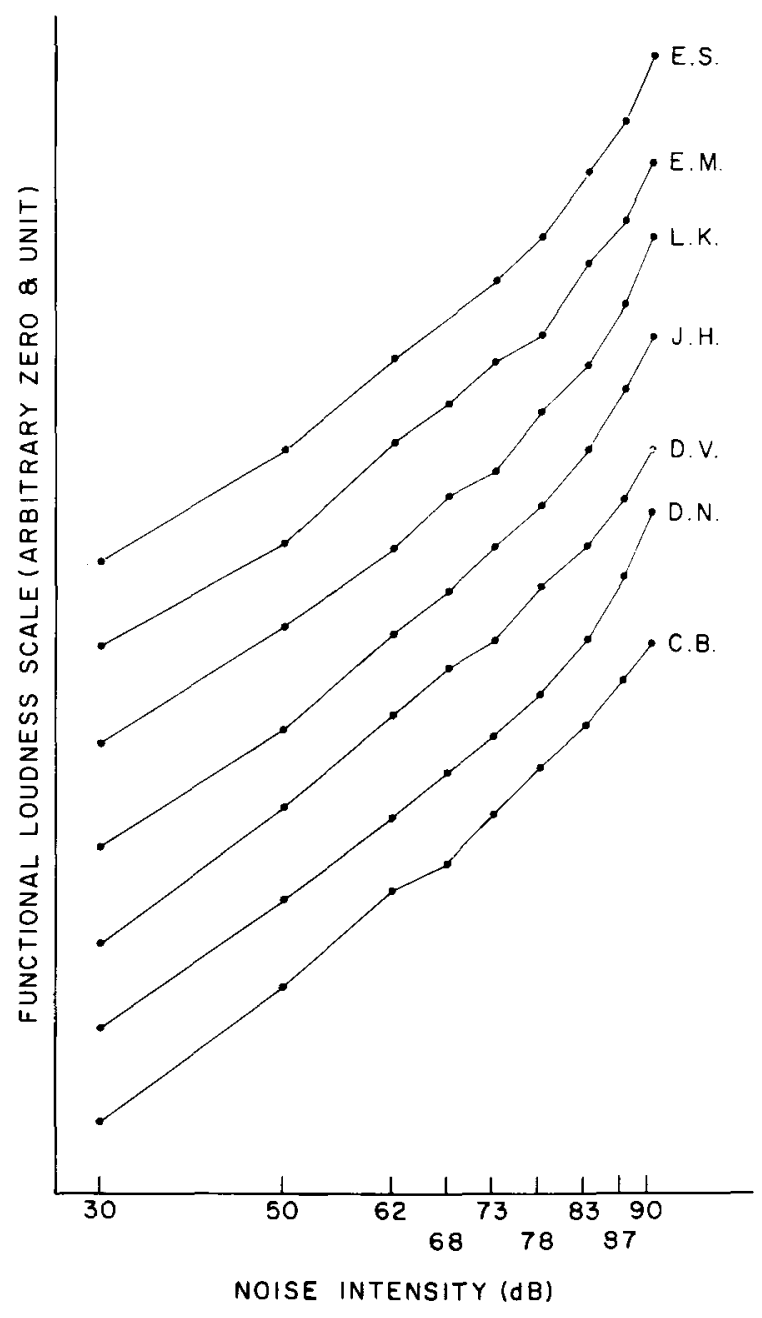

Figure 5. Functional loudness scale from bisection data for individual subjects.

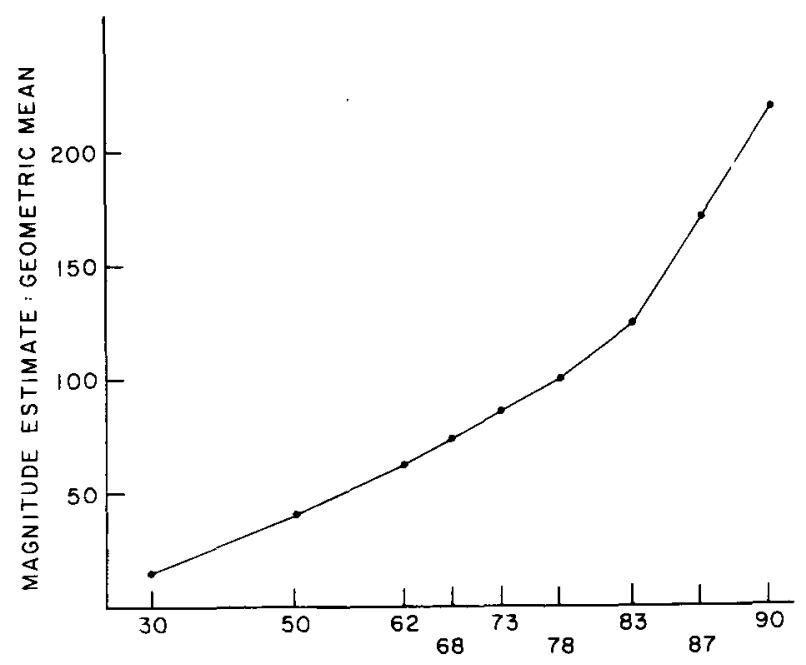

NOISE INTENSITY (dB): FUNCTIONAL SCALE SPACING

Figure 6. Magnitude estimates of loudness plotted against functional loudness scale. around half the size of exponents obtained from magnitude estimation (Marks, 1974b; Stevens, 1974). The present disagreement in loudness scales is thus entirely consistent with previous work.

Collateral evidence on this disagreement is given by the present judgments of the single stimuli. The present ratings of the single stimuli were approximately linear as a function of bisection loudness. However, the magnitude estimations were markedly nonlinear, as shown in Figure 6. Considered as a function of sound pressure, the present magnitude estimation data yielded a power function exponent of .59 , quite close to the cited standard value of .6. These results also agree well with previous work (but see footnote 6).

Finally, Figure 7 plots Garner's lambda scale as a function of the present bisection scale. This comparison must be interpreted cautiously because Garner used a $1,000-\mathrm{Hz}$ tone measured in phons and the present work used a noise measured in decibels. Nevertheless, the curve is reasonably straight except for a moderate nonlinearity at the two lowest intensities. This discrepancy may be a consequence of Garner's fractionation analysis in which inconstancies in the fractionation ratio would have cumulative effects toward the lower intensities. Over the main range, however, the agreement seems fairly good.

\section{Order Effects}

Two kinds of order effects arise in loudness bisection. The first may be called sensory fading, reflecting the fact that the most recent of a sequence of equally intense sounds is loudest. If all three sounds in the bisection sequence have equal intensity, they will not be equally loud; the middle stimulus would be a proper bisector only if loudness faded proportionately with time. Since the loudness fading is largely immediate, it follows that the bisector must be set at a higher decibel value than that of two equal bisectees.

The second kind of order effect reflects unequal weighting of the two stimuli, such as can occur in any task of serial integration (Anderson, 1974c, Section 6). The two bisectees may be unequally weighted so that the bisector is not at their midpoint, but at some other partition point, the $40: 60$ point, for example.

The two order effects are conceptually different. The first is represented in terms of sensation scale value, whereas the second is represented in terms of the weight parameter. Both order effects can be studied in the 4 by 4 subdesign in which the two bisectee stimuli have the same intensity levels. In this 4 by 4 subdesign, the sensory order effect appears in the comparison of the diagonal entries to the physical value. The weighting effect appears in the comparison of corresponding entries above and below the diagonal.

The top half of Table 1 lists mean decibel values for the bisector response. The second entry in the top 


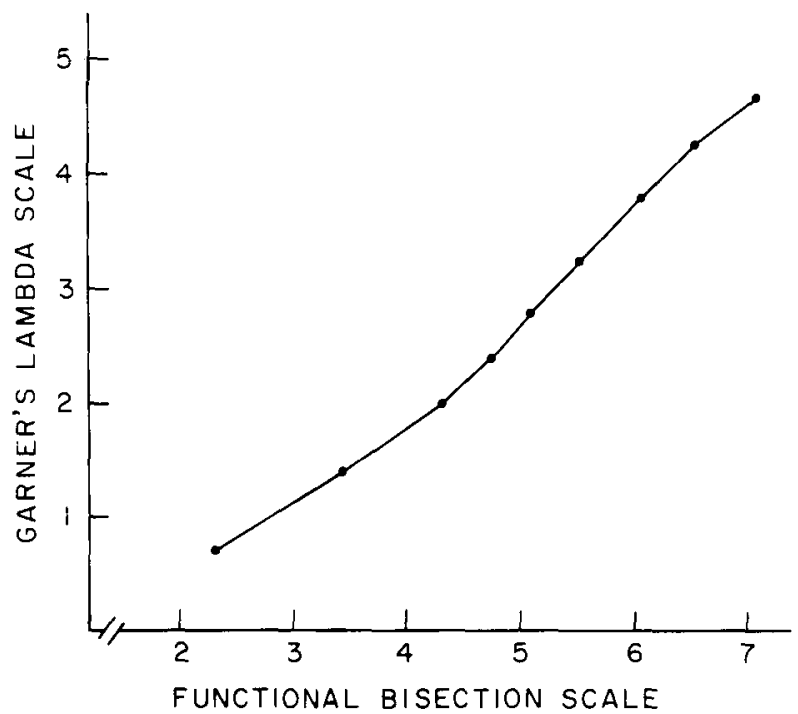

Figure 7. Garner's lambda scale of loudness plotted against functional loudness scale (mean transformed response of Table 1 ).

row shows that the bisector of two $50-\mathrm{dB}$ sounds has the value of $52.1 \mathrm{~dB}$. The entries for the 68-, 78-, and $87-\mathrm{dB}$ pairs show similar, but progressively smaller, effects. Thus, the differences between bisector and bisectee are $2.1,1.8,1.4$, and $.6 \mathrm{~dB}$, respectively.

The progressive decrease in these four decibel differences does not imply a corresponding decrease in the size of the sensory order effect. A proper comparison must be made in terms of the loudness scale, not the physical intensity scale, which is, in this case, rather misleading. From Figure 4 and Table 1, the difference between 52.1 and $50 \mathrm{~dB}$ is approximately .86 on the bisection scale of loudness (arbitrary unit). Comparable values for the other three differences are $.72, .84$, and .62 . These four values are roughly equal. Present data thus support the hypothesis that this sensory order effect is constant in subjective magnitude.

The other order effect refers to unequal weighting of the first and second bisectees. For example, the upper half of Table 1 shows that the bisector of the 50-87 dB pair is $74.3 \mathrm{~dB}$, whereas the bisector of the $87-50 \mathrm{~dB}$ pair is $71.2 \mathrm{~dB}$. Thus, the bisector is closer to the second bisectee. That means that the w parameter in Equation 1 is greater than .50. Of course, estimation of the weights should be in terms of subjective loudness values from the lower half of Table 1 , and is more efficient when based on the marginal means of the 4 by 4 subdesign, as is done below. It is worth noting, however, that estimation based on decibel values gives nearly the same weights.

To estimate the weight parameter, the serial integration model may be applied in the usual way (Anderson, 1974a, p. 249). It is assumed (see also below) that a given stimulus has the same loudness value in first and second positions. It follows that the net loudness ranges of the row and column means are proportional to their respective weights. Let $w$ denote the relative weight of the second bisectee, so that 1 - w denotes the relative weight of the first, and let $s_{50}$ and $s_{87}$ denote the loudness values of the smallest and largest bisectees. Then, from the lower half of Table 1,

$$
\mathrm{w}\left(\mathrm{s}_{87}-\mathrm{s}_{\mathrm{s0}}\right)=6.53-3.45=3.08
$$

and

$$
(1-w)\left(s_{87}-s_{50}\right)=6.28-3.64=2.64 \text {. }
$$

Solving yields $w=.54$. Thus, the second bisectee has somewhat greater weight than the first.

Both order effects were stable over subjects. The sensory order effect can be assessed for the four cases in which both bisectees have equal intensity, or a total of 28 cases over the seven subjects. In 27 of these 28 cases, the bisector had greater value than the bisectees. The mean decibel differences, averaged over the four cases for the individual subjects, listed in the top-down order of Figure 5 were $1.2,1.1,2.0,1.2,1.3,1.6$, and $2.2 \mathrm{~dB}$. The corresponding individual $w$ estimates were $.54, .52, .54$, $.57, .52, .56$, and .52 .

A few supplementary comments on these analyses may be added. First, and most important, the assumption that row and column stimuli have the same loudness values is more stringent than necessary. The same weight estimates are obtained if loudness values differ by a constant between row and column. Since the first order effect was approximately constant, its presence does not affect the weight estimate for the second order effect. Second, the weight estimate could be made more reliable by using the slopes for the four row and column means rather than just the net range between the smallest and largest means. Third, the possibility that these order effects might be sensitive to temporal intervals was checked in a supplementary run in which the time intervals between the three stimuli in a bisection sequence were increased from .5 to $1.0 \mathrm{sec}$. Four of the original subjects were recovered, and each was run in 10 replications of the 4 by 9 design. Results were much the same for both order effects. The weight estimate, in particular, was $\mathrm{w}=.53$.

The present results are consistent with a negative time-order effect with lifted weights (Anderson, 1971). That study tested various explanations of a recency effect that is typically obtained in serial psychophysical averaging. The results indicated that the time-order effect was not a change in scale value, and suggested an interpretation in terms of a weight 
parameter. A related view has been developed by Hellström (1978) in studies of duration and loudness. In this weighting interpretation, the misnamed "hysteresis" effect has a natural representation as a weight parameter.

Finally, it may be emphasized that the present method provides a loudness scale that is free of order effects. Averaging over both orders of presentation is not necessary because the scale is constructed from the marginal means of the second factor of the design. The weight parameter is automatically included in the unit of this scale. For scaling purposes, this procedure has certain advantages to counterbalancing in a square design.

\section{Previous Work on Bisection}

Detailed comparison of present and previous bisection results is difficult for a variety of reasons. However, one or two comparison values are worth noting.

From Garner's (1954a) Figure 6, the bisector of a 50-90 dB pair appears to be slightly under $76 \mathrm{~dB}$, whereas the present Table 1 lists $77.3 \mathrm{~dB}$. However, since Garner's data were averaged over both orders of presentation, the present value must be corrected for the order effect. From the two 50-87 dB pairs, the order effect is estimated as $3.1 \mathrm{~dB}$, a value that may also be applied to the 50-90 dB pair. Half of this is therefore to be subtracted from the listed value to yield $75.7 \mathrm{~dB}$, quite close to that of Garner. At lower intensities, of course, the present bisection values run somewhat lower than Garner's, as indicated in the discussion of Figure 7.

Stevens (1955) states that his bisection data disagree with those of Garner and are only slightly less than would be predicted on the assumption that $10 \mathrm{~dB}$ represents a $2: 1$ change in loudness. (That is equivalent to a power function exponent of .6 as a function of sound pressure.) On the implicit assumption that the simple bisection model holds, this prediction would yield 80.8 as the bisector of the $50-90 \mathrm{~dB}$ pair. The corresponding empirical value from Stevens' Table $\mathrm{V}$ would be perhaps $80 \mathrm{~dB}$. That is substantially larger than the present value or that of Garner. Unfortunately, Stevens presented only "sample results," and his experiment apparently remains unpublished.

\section{Averaging and Differencing}

Results from the averaging and differencing tasks were unexpected and disappointing. The factorial graphs of Figure 8 do not show the same pattern as the bisection data. In the averaging data, the three lower curves are bunched together; in the differencing data, the curves deviate from parallelism, showing greater vertical spread in the middle than at either end. Group analyses showed signifi-

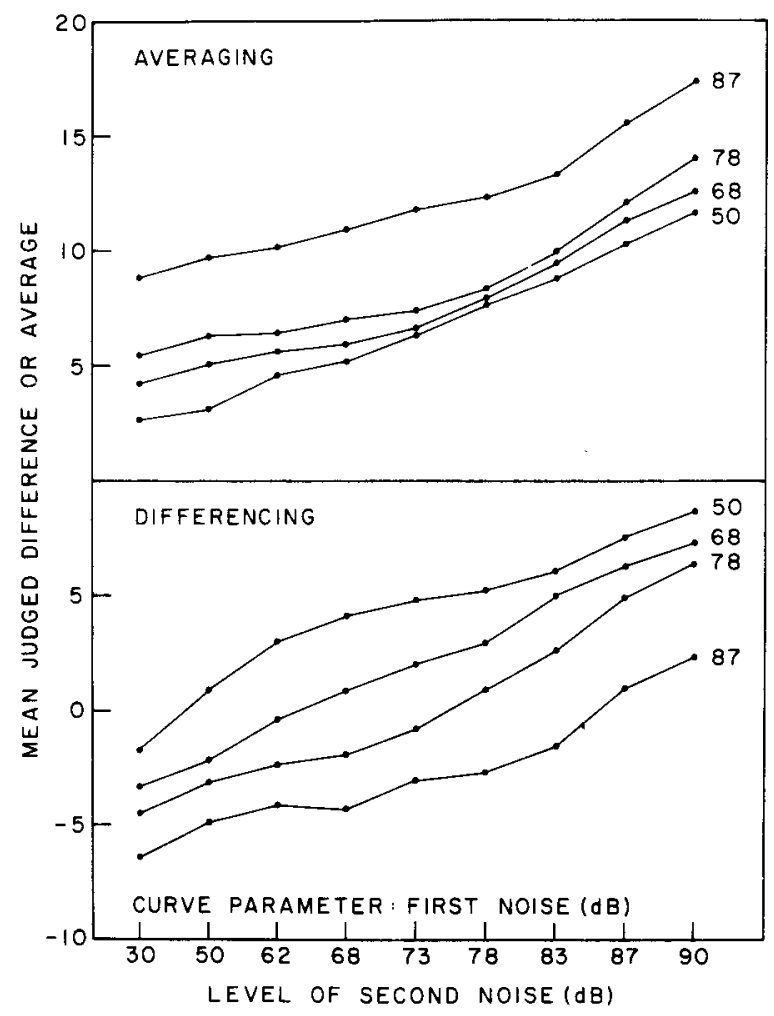

Figure 8. Factorial graphs of mean judgment of average loudness and difference in loudness, Experiment 2.

cant deviations from parallelism in both tasks, $\mathrm{F}(24,144)=1.85$ and 1.89 , respectively. Although these deviations are marginally significant, the pattern of deviations in Figure 8 indicates that the linear model does not provide a good account for the raw data.

A natural suspicion is that the deviations from parallelism reflect nonlinear use of the response scale. For the differencing task, in particular, the observed barrel pattern suggests that unit steps had lesser subjective value near the center of the response scale. If the deviations result only from nonlinear response, then the data could be made parallel by monotone transformation. Accordingly, monotone transformations were applied to make each subject's data maximally parallel in the same way as for the bisection data (see below). Instead of clearing up the discrepancy, however, this procedure accentuated it. In the group analyses, the interactions increased to $F(24,24)=$ 2.54 and 4.40 in the two respective tasks.

This increase in significance level may seem odd because the transformation naturally makes the data much more parallel. At the same time, however, the transformation also decreases the individual differences that enter into the group test, as well as smoothing the random error that enters into the individual tests. The differencing model fared worse 
than the averaging model in these individual tests: five of seven individuals showed highly significant deviations from the differencing model compared to one of seven for the averaging model. Overall, these analyses indicate that the nonparallelism should not be attributed to response nonlinearity but reflects flaws in the models, especially for the differencing task.

This outcome was unexpected, not so much for the statistical significance of the model discrepancies as for the disagreement in data patterns between the averaging and differencing tasks, on the one hand, and the bisection task, on the other. All three tasks had yielded similar patterns and scales in previous work on grayness (Anderson, 1976). Further, the bisection data were mainly consistent with the averaging and differencing data from the present Experiment 1. Thus, the question seems to be why the averaging and differencing models worked reasonably well in Experiment 1 but poorly in Experiment 2. General procedure was essentially the same in both experiments, but in Experiment 2 there was an increase from 5 to 9 in the number of noise levels and an increase from 40 to $60 \mathrm{~dB}$ in the stimulus range. Both factors are known to cause difficulty for absolute identification. Accurate discrimination becomes increasingly difficult as the number of stimuli increases beyond seven (Miller, 1956). Identification also becomes increasingly difficult when the stimulus is farther from an anchor (Berliner, Durlach, \& Braida, 1977; Weber, Green, \& Luce, 1977).

Averaging and differencing both require some preliminary valuation operation (Anderson, 1977b, Figure 1) that yields subjective magnitude for the individual stimuli. This valuation operation may be considered as analogous to absolute identification, and so would be made more difficult by increases in both stimulus range and number of stimulus levels. From this standpoint, the bunching of the three lower curves in the averaging data of Figure 8 suggests a tendency to simplify by dichotomization, with the first noise being treated as soft or loud in order to concentrate on the second noise. The barrel shape in the differencing data could reflect similar dichotomization tendencies.

It also should be noted that the large number of judgments required of the subjects could have contributed to such tendencies to simplify the valuation operation. Some suggestion of this appeared in Experiment 1 , in which two subjects exhibited semidichotomization in Part 2, as discussed previously.

These considerations point to the need for further methodological analysis of the averaging and differencing tasks. From a perceptual standpoint, indeed, these tasks seem somewhat artificial. Tasks that involve more perceptual kinds of integration, such as bisection and various illusions (Anderson, 1974a;
Clavadetscher \& Anderson, 1977), deserve more attention, both as a basis for measurement and for inherent perceptual interest.

\section{Related Work on Loudness Averaging}

Two other reports have considered loudness averaging. Parducci, Thaler, and Anderson (1968) found marked deviations from parallelism for judgments of average loudness of four noise bursts, rated on a 6-point scale. Later work (e.g., Parducci, Note 1) has made it increasingly clear that rating scales with under 10 response categories run serious risks of response bias. These data therefore have doubtful bearing on the averaging model. The recency effect that was obtained is still valid, however, and agrees with the second order effect discussed above.

Curtis and Mullin (1975) found deviations from parallelism for average loudness of two tones, judged with a magnitude estimation response. Although not statistically significant, the nonparallelism was substantial and systematic. The factorial graph exhibited the diverging pattern that would be expected if the magnitude estimate was a nonlinear, accelerated function of loudness, as in Figure 5 above.

To rectify the magnitude estimation response, Curtis and Mullin applied a monotone transformation to make their data as parallel as possible, and used these data in further scaling analyses. Unfortunately, Curtis and Mullin did not obtain a test of goodness of fit, either for the monotone analysis or for their two-stage model based on power functions. Moreover, their 6 by 6 design is probably too small when the monotone transformation is based on a single score in each cell (see below). These analyses thus have limited bearing on the averaging model. The scaling results reported by Curtis and Mullin may be correct, of course, but they rest on the assumption that the averaging model was correct (see also Anderson, 1974b, p. 230; 1977b, p. 208).

\section{Related Work on Loudness Differencing}

Stevens (1971, Figure 4) discusses work of Beck and Shaw (1967) and of Dawson (1971), who obtained magnitude estimations of difference in loudness for pairs of tones. Both studies showed that these judgments were not linearly related to Stevens' sone scale. Further, Dawson found that the magnitude estimation of the difference did not equal the difference between the magnitude estimations of the separate tones. These disagreements were surprising, because the response procedure in both studies, namely, magnitude estimation, was supposed to provide the true measure of sensation.

Stevens resolved this disagreement by claiming that the difference task itself, despite the use of the magnitude estimation response, caused a bias. 
Indeed, this bias was identified with the "partition" bias that Stevens claimed was present in the ordinary rating response. An alternative interpretation is that the bias is in the method of magnitude estimation.

Support for this alternative interpretation was suggested by the observation (Anderson, 1972, p. 393, 1974c, p. 272; Marks, 1978) that the curves in Stevens' Figure 4 were roughly parallel-on a log response scale. By virtue of the parallelism theorem, that suggests that the difference model was correct, that magnitude estimation is biased, and that an approximate log transformation is needed to eliminate the bias.

Four other studies of loudness differencing have all relied on monotone transformation of the response. The first three used $101,200-\mathrm{Hz}$ tones, ranging from 50 to $104 \mathrm{~dB}$. The 45 possible pairs of these 10 tones form a triangular design that provides constraint needed for determining the best monotone transformation. Parker and Schneider (1974) obtained magnitude estimates of loudness differences; Schneider, Parker, and Stein (1974) obtained binary choices of the louder of two such loudness differences; Schneider, Parker, Farrell, and Kanow (1976) obtained magnitude estimates of the ratios of two loudness differences as well as the corresponding binary choices. In the fourth study, Birnbaum and Elmasian (1977) used a functional measurement approach to analyze ratings of differences and magnitude estimates of ratios of loudness of $1,000-\mathrm{Hz}$ tone pairs whose intensity varied from 42 to $90 \mathrm{~dB}$.

All four of these studies appear to add to the consensus about the loudness function. The first three studies reported exponents of $.26, .26$, and .23 , respectively, for a power function fit. The fourth study did not report an exponent, but visual inspection of the graph seems to indicate a comparable value.

Unfortunately, it must be added that the first three studies suffer from two problems. The first problem is the lack of a test of goodness of fit. These studies rely on stress-correlation-scatterplot statistics that are seriously inadequate for testing models (Anderson, 1977b; Anderson \& Shanteau, 1977). This point may be illustrated with the difference judgments in the lower panel of Figure 8. The proportion of variance accounted for by the interaction, which is analogous to the stress statistic, is .024 . If the differencing model is fit to these data, the correlation between predicted and observed is $\mathbf{9 8 8}$. Judged by these statistics, it would seem that the differencing model fit the data extremely well. These statistics conceal the substantial nonparallelism that is at once apparent in the factorial graph of Figure 8.

The second problem is more serious, since it concerns the validity of the transformation procedure. In the cited studies, the transformation appears to be based on a single mean or central value in each cell of the design. That does not provide adequate constraint on the transformation, as indicated below. As a consequence, real nonadditivities in the differencing task may be incorrectly attributed to nonlinearities in the response scale and improperly eliminated. The scaling results of these studies may be correct, of course, but they rest on essentially untested and insecure assumptions that the differencing model is actually correct. ${ }^{2}$

The functional measurement analysis employed by Birnbaum and Elmasian (1977) is more satisfactory on both of these problems. Replications was treated as a third factor in the MONANOVA analysis so that the monotone transformation was based on 10 replications for each subject. Only a single monotone transformation was used, so that the error terms cited in this paper do not provide a strictly proper test of fit. That is probably not a serious problem, however, since the factorial graphs showed little deviation from parallelism. Furthermore, the present results suggest that their use of 10 replications may have provided adequate constraint on the monotone transformation. Nevertheless, the question of constraint and power remains a serious problem for all attempts to use nonmetric analyses, as discussed below.

\section{Application of Monotone Transformation}

There are three major problems in monotone transformation analysis. The first two concern how to calculate a best transformation and how to test whether it is good enough. For linear models, a solution to the first problem has been obtained in recent developments in numerical analysis (de Leeuw, Young, \& Takane, 1976; Kruskal, 1965). A solution to the second problem has been obtained in recent applications of functional measurement (Anderson, $1975,1977 b$ ) that provide a general error theory for nonmetric analysis. These were employed as follows.

The ADDALS program of de Leeuw et al. was used here. It employs much the same procedure for monotone regression as Kruskal's MONANOVA but has the particular advantage of transforming the entire distribution of responses in each cell rather than only their mean. The least squares option was used, together with a criterion of .001 for improvement of stress on one iteration, except that a criterion of .0005 was used for the main analysis of the differencing data, as noted in footnote 3 .

This program was applied separately to the first five and to the second five replications for each subject for each of the three tasks of Experiment 2. Two independent estimates of the best monotone transformation were thus obtained for each subject for each task. With independent estimates, a valid test of goodness of fit is possible.

The question is whether reliable nonadditivities remain in the transformed data. To test this, the additive model was fit separately to the mean values of each set of five replications of transformed data. 
These fitted values were then subtacted from the given mean values to yield one row by column matrix of deviation scores for each monotone transformation. The null hypothesis that the transformed data are additive implies that the row by column interaction in the matrix of deviation scores is zero. If the monotone transformation has been successful, therefore, the mean square for row by column interaction should be nonsignificant. A valid error term for this test is provided by the mean square for the row by column by transformation interaction. For the 4 by 9 design, these individual analyses yielded $F$ ratios on $(24,24)$ df that have been cited above. Group analyses were obtained in the usual way by treating subjects as a random factor, and these tests were also cited above.

The need for proper tests of goodness of fit may be reemphasized by a result from a previous study of length bisection (Anderson, 1977a). These data yielded a correlation between predicted and observed of .9997 despite the presence of significant and moderately substantial discrepancies from additivity. This is only one illustration of the fact that correlations and scatter plots are insensitive to real deviations from theoretical models. Significant discrepancies need not cause rejection of the model, of course, but they do need to be brought out in the open.

\section{Cautionary Note on Monotone Analysis}

The third major problem in monotone analysis concerns the need for protection against improper transformation. Monotone transformation gives tremendous flexibility in fitting the data to the model. The transformation may be overly obliging. It may force data to seem additive even when they come from an inherently nonadditive process. Unless the design contains adequate constraints, it will not have adequate power to detect real nonadditivity. Without adequate power, the analysis rests on mere assumption that an additive model holds.

Almost nothing is known about this question of power. In principle, factorial design can provide adequate constraint. In practice, the factor levels may have to be very numerous and very close together to do the job.

One possible way to increase constraint is to obtain multiple replications (for a given individual). The entries in each cell of the design then form a little distribution, and the transformation would be applied to the entire distribution rather than to just the mean or median. Because these distributions overlap across cells, they should provide greater constraint than their mean or median.

Some relevant results from the present study will be presented here, together with unpublished results from previous studies of bisection. These results lead to two main conclusions. First, it seems quite clear that a single score in each cell of the design does not ordinarily provide adequate constraint, even when this score is a mean or median of a number of replications. Second, more tentatively, it appears that adequate constraint can be obtained with the procedure of multiple replications.

The first result comes from a study of grayness bisection (Anderson, 1976) in which each subject served in 10 replications of a 5 by 6 factorial. Each subject's data were analyzed in four ways: 10 monotone transformations, each for a single replication; 5 monotone transformations, each for 2 replications; 3 monotone transformations, each for 3 replications; and 2 monotone transformations, each for 5 replications. The most directly relevant quantities are the error mean squares: These had average values over subjects of $.0024, .0048, .0064$, and .0061 , respectively.

These error mean squares are the row by column by transformation interactions. If transformation makes the data more additive than is proper, then this error term will be artificially low. Accordingly, the rapid increase over the first three error terms provides a strong warning that one or two replications do not provide adequate constraint. At the same time, the rough equality of the last two error terms suggests that three replications may be adequate. The present use of five replications was based on this and other similar evidence. This evidence is not conclusive, because there would presumably be a tendency for the error term to increase with number of replications merely from increases in sample range. It may be doubted that such increases would be substantial, but the question has not been investigated in detail.

More cogent evidence is available when the model is incorrect. In that case, the $F$ ratio for row by column interaction provides an indication of power. For length bisection (Anderson, 1977a), the fivereplication method yielded significant discrepancies from parallelism. That is prima facie evidence that the data are inherently nonadditive, and that the fivereplication method can provide adequate power.

This F-ratio approach was applied to the differencing data of the present Experiment 2. When the data were transformed one replication at a time, the average error mean square was .0412 and the mean individual $\mathrm{F}$ for deviations from additivity was only 1.49. When the data were transformed five replications at a time, the average error mean square increased to .0560 , yet the mean $F$ also increased markedly to 3.42 , and five of the seven subjects showed significant interactions. ${ }^{3}$

This last result has basic importance for monotone analysis. The results from five-replication transformation indicate that the differencing model is wrong. Hence, the low $F$ ratios obtained with onereplication transformation imply that one replication per cell does not provide adequate power.

Two related results on pooled group data are also of interest. When the mean differencing data of Figure 8 were subjected to monotone transformation, 
they became almost perfectly parallel. Stress was .0029 and the proportion of variance accounted for by the interaction was .00001 . Essentially the same result was obtained by transforming the means of the individual data given by the five-replication transformation. In both cases, monotone transformation made the pooled group data appear additive, even though substantial and real nonadditivities were present in individual data.

The above results indicate that monotone analysis is far more demanding than has been recognized. It is apparent that procedures in common use do not provide adequate power to validate the basic model on which the transformation depends. However, the procedure of transforming a number of replications simultaneously does show provisional promise to provide adequate power for monotone analysis.

More systematic work on this problem of power is necessary. Systematic studies of the multiple replication method are needed for artificial data, especially with nonadditive models. Other approaches should also be studied, including the criteria of stimulus and response generality, and the use of twooperation models (Anderson, 1977b, pp. 210-211).

Two-operation models provide an opportunity to bypass the main problem with monotone analysis. The essential idea is to use one operation as the frame for monotone transformation and the other operation for testing goodness of fit. In the models $\mathrm{A}+\mathrm{B}+\mathrm{C}$ and $\mathrm{A}+\mathrm{B} \times \mathrm{C}$, for example, the response could be transformed to minimize the $\mathrm{AB}$ interaction. The $B C$ interaction would then provide a model test that is independent of the monotone transformation. This approach is more demanding in that two integration operations are involved. Where it is applicable, however, it would be expected to provide a simple, powerful basis for monotone analysis:

\section{DISCUSSION}

Modern studies of loudness measurement begin with the work of W. R. Garner and S. S. Stevens in the mid-1950s. Garner attempted to employ the physical response methods of fractionation and bisection; Stevens began with fractionation but shifted to the verbal response method that he called magnitude estimation. These two approaches at once produced conflicting results (Garner, 1954a, 1954b; Stevens, $1955,1957)$. In the intervening time, Stevens' approach has flourished while Garner's approach has fallen into neglect. The present work supports Garner, both in result and in principle, and suggests that subsequent reseach took the wrong path.

The central problem is that of validity or, more precisely, that of obtaining a validational base. Magnitude estimation faces the obvious objection that the responses are mere number-words, lacking legitimate claim to be true numbers on a linear scale of sensation. Fractionation faces the objection that the outcome can depend heavily on contextual factors. Both Stevens and Garner attempted to provide a validational base.

\section{Magnitude Estimation}

Stevens faced the validity problem in his initial paper on magnitude estimation of loudness. "Use various standards," advised Stevens, "for it is risky to decide the form of a magnitude function on the basis of data obtained with only one standard" (1956, p. 6). Had different standards yielded the same function, that would have provided some validational support. But, in fact, different standards can yield markedly different results-as shown in Stevens' own Figure 4. This and related effects have been verified by numerous subsequent investigators. Stevens' later precept of using no fixed standard of course eliminates this basis for validation.

An alternative basis for validation could be sought from integration tasks involving sum and difference judgments, for example, or fractionation and bisection. Results from these tasks have generally disagreed with magnitude estimation. Stevens, however, has repeatedly claimed that none of these tasks constitutes a proper validity criterion (Marks, 1974b, p. 257; Stevens, 1974; see also above discussion of Beck \& Shaw, 1967).

One major base for validation remains, namely, cross-modality matching. However, it has long been recognized (Attneave, 1962; Ekman \& Sjöberg, 1965; Treisman, 1964; Zinnes, 1969; see also Anderson, 1972, 1974a; Krantz, 1972) that scales from crossmodality matching are, at best, unique only up to a power transformation. Even if it is assumed that cross-modality matching is empirically true, therefore, that still leaves magnitude estimation without a validational base. ${ }^{4}$

\section{Fractionation and Bisection}

Garner also faced validity problems. At that time, the method of fractionation relied on the assumption that subjects fractionated at the assigned ratio, whereas Garner (1954b) had found dramatic context effects on the effective ratio. Similarly, the method of bisection relied on the assumption that subjects responded at the true subjective midpoint, whereas order effects were actually obtained.

But Garner saw how fractionation and bisection could be combined to obtain a validational base. $\mathrm{He}$ assumed that fractionation judgments obeyed the fractionation model, though with some unknown fraction. Also, he assumed that bisection judgments were made at the true sensory midpoint. ${ }^{5}$ Under these assumptions, fractionation yields a $\log$ linear scale and bisection yields a linear scale, 
each with an unknown constant. If both procedures measure the same thing, as Garner explicitly assumed, then the two scales can be brought into linear relation by estimating the two constants. As Garner's (1954a) Figure 9 shows, this succeeded remarkably well.

This linear relation was Garner's validity criterion. He rightly observed that the linearity condition assesses the "validity of the entire procedure used," including all the cited assumptions. Garner's paper thus constitutes a landmark in the measurement of loudness.

Science contains no absolute validity criteria, of course, and Garner's was not airtight. His bisection and fractionation assumptions both require consideration. Garner noted that a linear relation could also be obtained if the equisection judgments were not equal but varied as a power of loudness. That possibility is less remote than Garner thought, since it can be shown that order effects, as are obtained with loudness bisection, will induce something resembling a power relation. However, Garner presented evidence that order effects were balanced out. Some bias could remain, owing to the nonlinear relation between intensity and loudness, for example, but that would seem to be negligible.

Garner's fractionation assumption is more problematical. It can be shown that his linear relation would also be obtained if the fractionation ratio was not constant but varied exponentially with successive fractionations. This implication follows from a peculiarity of the fractionation method (Anderson, $1974 a$, p. 271). Moreover, later analysis of Garner's data suggest that the fractionation ratio was, indeed, not constant (Anderson, 1974a, p. 270). Such nonconstancy in the fractionation ratio may be responsible for the nonlinearity at the low end of the curve in Figure 7.

These objections aside, the fact remains that Garner's analysis went to the heart of the problem. Although his results have received lip service, the methodological cogency of his approach has been neglected. The present approach is in the same spirit as that of Garner and gives similar results. ${ }^{6}$

\section{Nature of Sensation}

Both Stevens and Garner treated loudness as a unitary, conscious quality. This view seems reasonable, indeed quite traditional, but it may be too simple. Eisler (1965) and Treisman (1964) have suggested that two processing stages are involved, an initial stage that yields magnitude of simple sensation and a later stage for comparison or discrimination among two or more stimuli. Marks (1974a, Equations 1-2) adopted a similar view.

A more general view (Anderson, 1975, p. 479, 1976; see also Marks, 1978) recognizes that there are many stages between the observable stimulus and the observ- able response. Conscious sensation is a window on one stage of this sequence and often, if not typically, represents preconscious integration across more than one sense modality. It deserves consideration, therefore, as Eisler, Treisman, and Marks have suggested, that the methods of Stevens and Garner are not actually in conflict but measure at two different stages.

On intuitive grounds, this harmonious resolution seems unlikely. Subjects do not feel that they are judging different qualities when using category ratings than when using magnitude estimation. Yet the ratings, which agree with bisection, disagree sharply with magnitude estimations. Of course, introspection is not adequate. This difference between ratings and magnitude estimation merely reemphasizes the necessity for a validational base, whether to support the hypothesis of two or several scales or to decide among them.

A deeper aspect of this issue stems from the view of conscious sensation as an integrated resultant of preconscious sensory components. On this view, scaling at the stage of conscious sensation, which has been the concern of traditional psychophysics, largely ignores the deeper problems of sensation and perception. An adequate approach demands the development of methods and theory that can dissect conscious sensation into its components. Consciousness is not an ultimate but a fortunate peephole into the depths of perception.

This general view agrees well with Garner's conception of sensation as a theoretical construct:

Whatever the ultimate solution of the loudness scaling problem may be, it is clear that the problem is not a simple one. Certainly it is not as simple as asking somebody what sounds half, or a quarter, as loud as something else. The method used in these experiments is one step in the direction of thinking of a loudness function as a derived function (even as a hypothetical construct) which cannot be directly observed but which must be inferred (1954a, p. 87).

The present bisection scale of loudness, like that of Garner, is a hypothetical construct. Its validity rests on the bisection model from which it derives.

The general potential of algebraic models in perceptual analysis has been recognized by many investigators working on many different substantive problems. Modern measurement theory has brought a new level of practicability and precision to the use of such models. And, fortunately, experimental studies increasingly show the empirical validity of such models.

A major virtue of algebraic models is that they go beyond measurement. Measure is implicit in the structure of perception, and scaling is just a working tool for substantive problems of perceptual analysis and synthesis. Algebraic models do not solve all or even most problems of perception. Where they are applicable, however, they can provide a penetration not otherwise obtainable. 


\section{REFERENCE NOTES}

1. Parducci, A: Category ratings: Still more contextual effects. Paper presented at International Symposium on Social Psychophysics, University of Mannheim, Germany, October 10, 1978.

2. Anderson, N. H. Cognitive algebra and social psychophysics. Paper presented at International Symposium on Social Psychophysics, University of Mannheim, Germany, October 10, 1978.

3. Cross, D. V. On judgments of magnitude. Paper presented at International Symposium on Social Psychophysics, University of Mannheim, Germany, October 10, 1978.

4. Coleman, B. J., Graf, R. G. \& Alf, E. F. Assessing power function relationships in magnitude estimation. Paper presented at the meetings of the Western Psychological Association, San Diego, April 6, 1979.

\section{REFERENCES}

Anderson, N. H. Functional measurement and psychophysical judgment. Psychological Review, 1970, 77, 153-170.

ANDERSON, N. H. Test of adaptation-level theory as an explanation of a recency effect in psychophysical integration. Journal of Experimental Psychology, 1971, 87, 57-63.

ANDERSON, N. H. Cross-task validation of functional measurement. Perception \& Psychophysics, 1972, 12, 389-395.

Anderson, N. H. Algebraic models in perception. In E. C. Carterette \& M. P. Friedman (Eds.), Handbook of perception (Vol. 2). New York: Academic Press, 1974. (a)

ANDERson, N. H. Cross-task validation of functional measurement using judgments of total magnitude. Journal of Experimental Psychology, 1974, 102, 226-233. (b)

Ande RSON, N. H. Information integration theory: A brief survey. In D. H. Krantz, R. C. Atkinson, R. D. Luce, \& P. Suppes (Eds.), Contemporary developments in mathematical psychology (Vol. 2), San Francisco: Freeman, 1974. (c)

Anderson, N. H. On the role of context effects in psychophysical judgment. Psychological Review, 1975, 82, 462-482.

ANDERSON, N. H. Integration theory, functional measurement, and the psychophysical law. In H. G. Geissler \& Yu. M. Zabrodin (Eds.), Advances in psychophysics. Berlin: VEB Deutscher Verlag, 1976.

ANDERSON, N. H. Failure of additivity in bisection of length. Perception \& Psychophysics, 1977, 22, 213-222. (a)

Ande RSON, N. H. Note on functional measurement and data analysis. Perception \& Psychophysics, 1977, 21, 201-215. (b)

Anderson, N. H., \& Shanteau, J. Weak inference with linear models. Psychological Bulletin, 1977, 84, 1155-1170.

AtTneave, F. Perception and related areas. In S. Koch (Ed.), Psychology: A study of a science (Vol. 4). New York: McGrawHill, 1962.

BECK, J., \& SHAW, W. A. Ratio-estimations of loudness intervals. American Journal of Psychology, 1967, 80, 59-65.

Berliner, J. C., Durlach, N. I., \& Braida, L. D. Intensity perception, VII. Further data on roving-level discrimination and the resolution of bias edge effects. Journal of the Acoustical Society of America, 1977, 61, 1577-1585.

Birnbaum, M. H., \& Elmasian, R. Loudness "ratios" and "differences" involve the same psychophysical operation. Perception \& Psychophysics, 1977, 22, 383-391.

Carterette, E. C., Barnebey, A., Loveli, J. D., Nagel, D. C., \& Friedman, M. P. On-line computing with the HewlettPackard 2116B moving-head disk operating system. Behavior Research Methods \& Instrumentation, 1972, 4, 89-94.

Clavadetscher, J. E., \& Anderson, N. H. Comparative judgment: Tests of two theories using the Baldwin figure. Journal of Experimental Psychology: Human Perception and Performance, 1977, 3, 119-135.

Curtis, D. W., \& Mullin, L. C. Judgments of average magni- tude: Analysis in terms of the functional measurement and twostage models. Perception \& Psychophysics, 1975, 18, 299-308.

Dawson, W. E. Magnitude estimation of apparent sums and differences. Perception \& Psychophysics, 1971, 9, 368-374.

de Leeuw, J., Young, F. W., \& Takane, Y. Additive structure in qualitative data: An alternating least squares method with optimal scaling features. Psychometrika, 1976, 41, 471-503.

EISLER, $H$. The connection between magnitude and discrimination scales and direct and indirect scaling methods. Psychometrika, $1965,30,271-289$.

Ekman, G., \& Sjöве rg, L. Scaling. Annual Review of Psychology, $1965,16,451.474$.

GARNER, W. R. A technique and a scale for loudness measurement. Journal of the Acoustical Society of America, 1954, 26, 73-88. (a)

Garner, W. R. Context effects and the validity of loudness scales. Journal of Experimental Psychology, 1954, 48, 218224. (b)

Hellström, A. Factors producing and not producing time errors: An experiment with loudness comparisons. Perception \& Psychophysics, 1978, 23, 433-444.

KraNTZ, D. H. A theory of magnitude estimation and crossmodality matching. Journal of Mathematical Psychology, 1972, 9, 168-199.

Kruskal, J. B. Analysis of factorial experiments by estimating monotone transformations of the data. Journal of the Royal Statistical Society, Series B, 1965, 27, 251-263.

Luce, R. D., \& Galanter, E. Psychophysical scaling. In R. D. Luce, R. R. Bush, \& E. Galanter (Eds.), Handbook of mathematical psychology (Vol. 1). New York: Wiley, 1963.

MARKs, L. E. Stimulus-range, number of categories, and form of the category-scale. American Journal of Psychology, 1968, 81, 467-479.

Marks, L. E. On scales of sensation. Perception \& Psychophysics, 1974, 16, 358-376. (a)

Marks, L. E. Sensory processes. New York: Academic Press, 1974. (b)

Marks, L. E. Phonion: Loudness scales and the processing of auditory intensity. In M. J. Castellan \& F. Restle (Eds.), Cognitive theory (Vol. 3). Hillsdale, N.J: Erlbaum, 1978.

McGill, W. J. The slope of the loudness function: A puzzle. In H. Gulliksen \& S. Messick (Eds.), Psychological scaling. New York: Wiley, 1960

Mille R, G. A. The magical number seven, plus or minus two: Some limits on our capacity for processing information. Psychological Review, 1956, 63, 81-97.

Parducci, A., Thaler. L., \& Anderson, N. H. Stimulus averaging and the context for judgment. Perception \& Psychophysics, 1968, 3, 145-150.

Parker, S., \& Schneider, B. Nonmetric scaling of loudness and pitch using similarity and difference estimates. Perception \& Psychophysics, 1974, 15, 238-242.

Scheffé, H. The analysis of variance. New York: Wiley, 1959.

Schneider, B., Parker, S., Farrell, G., \& Kanow, G. The perceptual basis of loudness ratio judgments. Perception \& Psychophysics, 1976, 19, 309-320.

Schneider, B., Parker, S., \& Stein, D. The measurement of loudness using direct comparisons of sensory intervals. Journal of Mathematical Psychology, 1974, 11, 259-273.

Stevens, S. S. The measurement of loudness. Journal of the Acoustical Society of America, 1955, 27, 815-829.

Stevens, S. S. The direct estimation of sensory magnitudesloudness. American Journal of Psychology, 1956, 69, 1-25.

Stevens, S. S. On the psychophysical law. Psychological Review, $1957,64,153-181$.

Stevens, S. S. Issues in psychophysical measurement. Psychological Review, 1971, 78, 426-450.

Stevens, S. S. Perceptual magnitude and its measurement. In E. C. Carterette \& M. P. Friedman (Eds.), Handbook of perception (Vol 2). New York: Academic Press, 1974. 
Treisman, M. Sensory scaling and the psychophysical law. Quarterly Journal of Experimental Psychology, 1964, 16, 11-22.

Weber, D. L., Green, D. M., \& Luce, R. D. Effects of practice and distribution of auditory signals on absolute identification. Perception \& Psychophysics, 1977, 22, 223-231.

WEISS, D. J. Quantifying private events: A functional measurement analysis of equisection. Perception \& Psychophysics, 1975, 17, 351-357.

WEISS, D. J., \& Anderson, N. H. Use of rank order data in functional measurement. Psychological Bulletin, 1972, 78, 64-69.

Zinnes, J. L. Scaling. Annual Review of Psychology, 1969, 20, 447-478.

\section{NOTES}

1. Similar, but larger, deviations from parallelism in the monotonically transformed data were found in previous work on bisection of length (Anderson, 1977a). There it was incorrectly stated that a two-process model, including both an equating of sense distance and an equating of sense ratios, would also be monotonically additive and so could not account for the data. This differenceratio bisection model would be written $r=a\left(s_{1}-s_{2}\right)+b \sqrt{ } s_{1} s_{2}$. A theorem of Scheffé $(1959$, pp. 95f) shows that this model cannot be made additive by monotone transformation of $r$. However, this model implies divergence as stimulus magnitude increases, whereas the data showed convergence. Hence the conclusion remains valid that the composite difference-ratio model could not account for the data.

2. There is evidence that monotone analysis can reconstruct the stimulus metric from rank order data - when the basic model is known to be correct (e.g., Weiss \& Anderson, 1972). The present results indicate that monotone analysis will too easily make the data additive even when the model is incorrect. In that case, of course, the derived stimulus metric will not be valid.

3. For the five-replication transformation of the differencing data, the criterion was .0005 for improvement of stress on one iteration. This criterion gave virtually identical results to the .001 criterion, thereby showing that the failure of the differencing model was not due to insufficient convergence of the ADDALS program.

Strictly speaking, $F$ ratios are not comparable across the two conditions, because they are based on $(24,216)$ and $(24,24) \mathrm{df}$, respectively. To allow for the different $\mathrm{df}$, the mean Fs may be converted to $\mathrm{p}$ values, yielding $\mathrm{p}=.0724$ and .0019 for the oneand five-replication conditions, respectively. It is clear that transformation based on one replication had inadequate power to detect real discrepancies from the differencing model. It may be added that the difference between 216 and $24 \mathrm{df}$ for the denominator represents a rather small difference in power.

4. The fact that a power function often provides a good fit to magnitude estimation data is sometimes considered as validational support. However, Marks (1968) found that power functions also fit category rating data. Other evidence on validity of magnitude estimation is surveyed in Anderson (1974a, Note 2).

5. Strictly speaking, Garner used equisection, a more general task in which the given interval is to be divided into several equal parts. For present exposition, the distinction is not important.

6. Cautionary note on power functions. Exponents from power function fits are commonly used as descriptive statistics, and the present writers had assumed that they were useful for descriptive purposes, especially for comparing among different experiments. However, our experience in this work has raised doubt about the wisdom of relying on exponents to describe psychophysical data. One problem, of course, is that there is no agreement on either the propriety or the proper method of including threshold-type constants in the power function fit. What procedure is adopted can have substantial effects on exponent values. A second problem is that moderate changes in size of exponent have rather small effects on function shape, especially when the stimulus range is small. Exponent values are somewhat unreliable, therefore, and that makes it difficult to compare results from different studies when only mean exponent values are presented. Such comparisons have, perforce, been made in this paper, but although they seemed to work satisfactorily we feel that they should be interpreted cautiously. Certainly, it is more satisfactory to compare the actual data, as in the comparison of our results with Garner's in Figure 7.

The popularity of power functions stands in striking contrast to the dearth of studies of their psychometric properties. Unfortunately, the use of power functions as a descriptive statistic in curve fitting has been entangled with the use of magnitude estimation as a response measure. Recent work has begun to study the psychometric properties of power functions (e.g., Cross, Note 3; Coleman, Graf, \& Alf, Note 4), but much more needs to be done to establish appropriate uses and limitations.

(Received for publication December 21, 1978; revision accepted May 31, 1979.) 